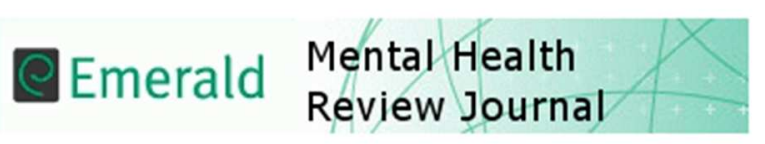

\title{
Do you want therapy with that? A critical account of working within IAPT
}

\begin{tabular}{|r|l|}
\hline Journal: & Mental Health Review Journal \\
\hline Manuscript ID: & MHRJ-11-2014-0044.R2 \\
\hline Manuscript Type: & Discussion Piece Review \\
\hline Keywords: & IAPT, CBT, Critique, Psychotherapy, NHS \\
\hline \multicolumn{3}{c}{} \\
\\
& $\begin{array}{r}\text { SCHOLARONE } \\
\text { Manuscripts }\end{array}$ \\
\hline
\end{tabular}


Do you want therapy with that? a critical account of working within IAPT

\section{Introduction}

Improving Access to Psychological Therapies (IAPT) is a programme that was conceived in 2004 as the ideological vision of one of the UK's most eminent clinical psychologists, David Clark and the economist, Lord Layard. This vision was realised in 2006 and rolled out in 2008. Although the overarching function was economic, in order to get people with anxiety and depression back into work, the IAPT programme dramatically re-shaped the provision of psychological therapies in the UK. Prior to IAPT, psychological therapy services were hindered by long waiting lists in secondary care services and brief counselling services in primary care that lacked consistency in terms of delivery. IAPT promised much and has delivered effective interventions to many who would previously been unable to access psychological therapy. However, from working within an IAPT service for nearly four years it has been difficult to maintain a belief that all is well with the system. The specific IAPT service I have worked in is not in itself substandard and is helping people with mental health problems. There are many committed and dedicated therapists delivering IAPT interventions, many of whom also acknowledge the limitations of the system but still endeavour to make real changes to people's lives. What I am going to put forward is a selection of fundamental misgiving with the practical implementation of the IAPT ideology and at times a critique of the ideology itself. This paper is presented by highlighting significant statements I have actually heard over the years or my interpretations of what has been said, followed by an analysis of the implications.

\section{“How can you work with someone if they don't have a diagnosis?"}

The IAPT framework can be considered a medical model of psychological therapy. Students on the one year therapy training scheme are taught a prescribed version of Cognitive Behavioural Therapy (CBT). The dominating theme is diagnosis and treatment. For example, if the client has difficulties in social situations, feels anxious and therefore avoids such situations or adopts other safety seeking behaviours, then they are assumed to have social anxiety and a protocol for treatment is administered. The students give a 'provisional' diagnosis (however in reality the word provisional is often dropped) and deliver the version of CBT they have been taught. Although adopting this medical model may have been 
necessary to secure funding for the IAPT programme ( $£ 700$ million to date) there are several ideological and methodological consequences that follow.

The first is self-explanatory: if psychological treatment is based on a diagnosis and associated findings from randomised control trials, this presupposes that psychiatric diagnoses are valid and reliable and that academic research samples can be compared to clinical populations; there are many who suggest that this is not the case, for example Kutchins and Kirk, (1999); Bentall, (2003). By assuming the validity and reliability of psychiatric diagnosis, therapists often lack a critical perspective and fail to see the client as an individual with individual problems. The training and expectations of IAPT therapists is dominated by diagnosis and associated manualised treatment. Case conceptualisation, the ability to formulate irrespective of diagnosis, is not realised. Working collaboratively with the client to develop a joint understanding of the problem and maintaining factors, and then working through them in a compassionate manner is not widely utilised within IAPT services. The reliance on diagnosis meant that I once heard: "My interventions are going well but I just can't seem to fit the client into the formulation". People are not a diagnosis; they do not seek help for their diagnosis. What people want is a safe place to be heard, to understand their difficulties and to develop ways of dealing with their problems. Often the experience in IAPT is multiple assessments, questionnaires to be completed at every session, a psychiatric label and a procession through a workbook of interventions.

Another issue is the practice whereby junior members of staff (Psychological Wellbeing Practitioners: PWPs) conduct the initial assessments, often via the telephone and in a very structured and brief manner. They follow the diagnostic ideology and try to collect symptoms, diagnose and then match the diagnosis to the relevant treatment. This is done purely for economic reasons, and is common in many IAPT services (Mind, 2010). Psychological assessment and treatment planning is an advanced and complex skill that takes many years of supervised practice to develop competence and eventually master. Although IAPT services are supposed to see clients with mild to moderate anxiety and depression, the reality is that often inexperienced members of staff without sufficient training or psychological knowledge are left trying to assess complex clients. Rather than seek advice or reflect on a complex assessment there is a tendency to make quick decisions and put clients on waiting lists. Then, after some months, when the client is seen for treatment the therapist finds that the recommended treatment is not appropriate for them. The impulsivity of the 
assessors is understandable given the context in which they work, where they may have to conduct eight of more of these assessments a day. There simply is not enough time for considered decision making and the necessary follow up such as obtaining existing psychiatric reports. From witnessing a room full of PWPs conducting telephone assessments the image of a 'therapy factory' springs to mind.

\section{"What's exposure? ...Oh you mean behavioural experiments"}

In most IAPT training institutions CBT is taught within a Beckian cognitive therapy framework. One reason for this is the theoretical leanings of the researchers teaching on the courses, another is the historical impact of cognitive therapy. Given that the courses only last a year it is understandable that there have to be omissions in the course content (perhaps this suggests that the courses are not long enough to cover what is necessary for effective practice). The whole third wave CBT movement has been excluded, apart from a cursory nod to Mindfulness. Acceptance and Commitment Therapy (ACT) and meta-cognitive approaches are growing in terms of theory and proven effectiveness and are considered to be the future of CBT; however these are not covered on IAPT courses. More importantly, the B of CBT is simply skimmed over and not given the attention it deserves. From a solid training in behavioural theory and related applications, therapists gain the ability to conduct functional analyses. From the findings of the analysis appropriate interventions such as behavioural activation (not just activity scheduling) and exposure can be implemented. One of the advantages of behavioural therapy is that it does not rely on psychiatric diagnosis; another is its proven efficacy when compared to pure Beckian cognitive therapy (e.g. Jacobson et al, 1996). When IAPT therapists are not trained in behavioural therapy they struggle to work with people with anything other than standard presentations of depression and anxiety amenable to Beckian cognitive therapy. When asked to name a behavioural intervention many IAPT therapists reply with behavioural experiments, a cognitive intervention that aims to test the validity of a belief or assumption.

\section{“Have you got the manual for working with poverty, social exclusion and a lack of hope?"}

This interpretation of the IAPT ideology refers more to the dominance of individual therapy, in particular CBT. Although CBT has a strong evidence base for its effectiveness, and other individual therapies are increasingly showing promise, many people are referred for help with problems that are best seen as arising from practical, systemic or community issues. Applying what is fundamentally a medical model to these issues is not helpful and can be seen as 
blaming the individual for their problems. Poorly trained or inexperienced clinicians can be guilty of applying inappropriate treatment protocols that are unlikely to be effective.

Seeing issues purely within a psychiatric framework can lead to missing potentially useful interventions: practical problems often have practical solutions. I witnessed an example of this when a therapist insisted on applying a CBT protocol for a client's anxiety despite the anxiety being a direct result of debt issues. My advice was that the client would be better off initially seeing a debt advisor. These concerns are reminiscent of Maslow's hierarchy of needs (Maslow, 1943). For example, asylum seekers need a degree of safety (leave to remain) before psychological therapy for trauma; housing issues need to be secure before interventions for worry; social isolation needs to be addressed before (or during) CBT with depressed clients. In addition, issues within families are better addressed by working with the family rather than with the individual in one to one therapy. I have worked with many clients who have problems because of the difficulties in their home life. Seeing them as an individual (which is the IAPT way) is not ideal. However, as IAPT services are usually distanced from secondary care mental health services, access to social workers, family therapists and generic health professionals is limited and all of the above issues are assumed to be addressed by GPs through a referral for CBT. In contrast, prior to IAPT, a referral would be sent to the local community mental health team and a wider range of interventions could be considered. This need for a greater range of interventions is supported by many organisations, for example the mental health charity, Mind (Mind, 2010).

\section{“Depression with panic attacks, moderate OCD and GAD: you got six sessions"}

Similar to many NHS services IAPT services are subject to the tendering process. Every five years providers have to compete in order to win the tender and deliver the service. This competition encourages providers to focus on their targets and has the unfortunate outcome that quantity comes before quality. Although IAPT was originally based on NICE guidelines I have heard on numerous occasions "NICE guidelines are just a guide". Managers reinterpret (or ignore) the NICE guidelines and set session limits in order to process more referrals in a timely manner. It is not uncommon for many IAPT services to set a limit of six sessions for CBT treatment, despite the fact that NICE guidelines specify many more, e.g. 16-20 for depression (NICE, 2009). Good clinical practice indicates that even more sessions are needed when dealing with co-morbidity. However, when quantity is prioritised above quality it is easier to hit targets. Reducing session limits from 12 to 6 for example, means that twice as 
many clients can be seen within the same time span. I have found that the more managers realise this the likely they are to reduce the sessions offered. There seems to be a rush for the lowest common denominator in attempting to win the tender. An outcome of this decrease in clinical standards is that clients will often not hit 'recovery' within the number of sessions offered and will leave the service unsatisfied (Mind, 2010). Many will be re-referred shortly after discharge. Thus, in many ways reducing the number of sessions is a false economy as clients will need to be seen again. However, services are also measured on number of referrals (the more the better) rather than individuals, so by reducing sessions you can both speed up the treatment time and increase the referral rate: two boxes ticked in one move. What is being forgotten by many services is the client experience. Having to go back for more treatment, because what was provided at first was inadequate, is unacceptable. I have heard of therapists working in such services discharging their clients after six sessions, midway through treatment of PTSD, and saying that they have to refer themselves back and go through the system again to continue with therapy, often meaning a delay of several weeks. Clearly tenders are being prioritised before clients. The good news though is that organisations like the $\mathrm{BABCP}$ are becoming aware of this practice and recently released a press statement outlining the case against reducing the number of session offered to clients (BABCP, 2014).

\section{“The tail wags the dog”}

This is actually what I have said myself on a number of occasions when Key Performance Indicators (KPIs) are discussed. IAPT services usually have experienced health professionals in senior roles and management. These team members have a thorough understanding of health services and recognise that the client experience is central to the system. Senior members of staff tend to make sound clinical decisions that aim to benefit clients. However, increasingly in IAPT (and other health services) there seems to be a move towards pleasing the commissioners through hitting targets rather than giving good clinical care. Although the targets are designed with the client experience in mind (for example referral to treatment targets), the services prioritise meeting the target rather than what the target represents. Designing and maintaining a system that gets clients from referral to treatment within a set amount of time requires a lot of planning and revision; hitting a referral to treatment target does not. For example, if you change what 'treatment' actually means then it's easier to hit the target. Everybody working in IAPT understands that 'treatment' means starting the psychological therapy. However many services interpret this differently in order to make the 
KPIs look good. I have witnessed services spending time discussing how to use the IT system to meet the targets. If you have two sessions where certain clinical measures are taken (called the minimum data set) then this can mean assessment and treatment according to the IT system, thus hitting the target for wait times. You can, therefore, arrange to have an assessment and then a follow up assessment. The computer system will interpret this as matched sessions and will assume the client has begun treatment. This is a blatant example of fudging the statistics, which is used in many IAPT services. In view of the pressures associated with re-tendering, this would be excusable up to a point if people were honest. However what I have witnessed is people believing their own bullshit. They actually convince themselves, and others, that a brief 10 minute follow up telephone conversation constitutes treatment: "Well they have been listened to, that means they have had some therapy". As well as being considered fraudulent this practice also has a hidden impact on clients who are left on increasingly long waiting lists for real treatment for their problems.

\section{Conclusion}

Ritzer, (1993) coined the phrase 'McDonaldization' when referring to contemporary western social life; Strawbridge and Woolfe, (2012) expanded on this theory when describing how the complexity of therapy is minimised when there is a focus on diagnosis and subsequent training in technique and manualised treatments. IAPT is a prime example of how this McDonaldization process is rolled out on a national scale: clients are put through the system and therapies are delivered and outcomes measured; everything is supposed to be manualised to the point of the idea of the 'interchangeable therapist'. I have on a number of occasions been asked to continue therapy with a client when the previous therapist has left the service prematurely. On these occasions there has been an expectation that I am able to simply continue where the last therapist finished (e.g. "they've had eight sessions for social anxiety already, you've got two left to complete the CBT and do the relapse prevention and blueprinting"); I have never agreed with this and have always seen the client as if they are presenting for the first time and facilitated our therapy within a more collaborative approach. However, the reality of the McDonaldization process is that some of the 'franchises' operate quite differently. Some (including the service I have worked at) struggle to deliver quality over quantity, whereas others embrace the process and emphasise re-tendering and often profit making. 
The main concern I have with the current direction of IAPT is how CBT and IAPT are now synonymous with each other; the IAPT version of CBT is being seen as all CBT can be. There is a risk of if the IAPT project fails then the reputation of CBT will be damaged. I am trying to put forward that the IAPT version of $\mathrm{Cbt}^{1}$ is not CBT.

Given all that has been put forward the question 'should IAPT be abandoned?' has to be asked. My response to this would be definitely not, as we would be throwing the baby out with the bath water. As stated earlier the creation of IAPT has benefited many people that would simply not have had a service before. However, the practical implementation of the project in many areas has failed. The blinkered reliance on the medical approach and the corrupting influence of the tendering process necessitates an overhaul of IAPT practises. Individual practitioners working in IAPT services can be mindful of the pitfalls and do what they can to avoid bad practice, but what is really needed is change at the governmental policy level and for this to filter down to the commissioners and finally to the services delivering the interventions. People of influence such as health ministers need to hear from both IAPT service users and clinicians about their experiences of IAPT; whether this is best addressed through academia is another question.

\footnotetext{
${ }^{1}$ Intentional mis-abbreviation
} 


\section{References}

BABCP, (2004). 'Bullying Culture' In NHS Mental Health Services Putting Vulnerable

Patients At Risk. British Association of Behavioural and Cognitive Psychotherapies Board Issue $25^{\text {th }}$ September 2014. www.babcp.com/About/Press-Releases.aspx

Bentall, R.P. (2003). Madness Explained: Psychosis and Human Nature. London: Penguin.

Jacobson, N.S., Dobson, K.S., Traux, P.A., Addis, M.E., Koerner, K., Gollan, J.K., Gortner, E. and Prince, S.E. (1996). A component analysis of cognitive-behavioural treatment for depression. Journal of Consulting \& Clinical Psychology. 64: 295-304.

Kutchins, H. and Kirk, S.A. (1999). Making Us Crazy: DSM-The Psychiatric Bible and the Creation of Mental Disorders. London: Constable.

Maslow, A.H. (1943). A Theory of Human Motivation. Psychological Review 50: 370-96.

Mind (2010). We Need To Talk: Getting the right therapy at the right time. London: Mind Publications.

National Institute for Health and Clinical Excellence (NICE) (2009), Depression: The Treatment and Management of Depression in Adults. National Institute for Health and Clinical Excellence, London, available at: www.nice.org.uk

Ritzer, G. (1993) The McDonaldization of Society. London: Pine Forge.

Strawbridge, S. and Woolfe, R. (2010). Counselling psychology: Origins, developments and challenges. In: Woolfe, R., Strawbridge, S., Douglas, B. and Dryden, W. (Eds.), Handbook of counselling psychology (3rd ed., pp.3-22). London: Sage. 